\title{
The Virtual Hospital
}

\author{
Authors: Kruti Patel, ${ }^{\mathrm{A}}$ Bahman Shokouhi, ${ }^{\mathrm{B}}$ Edward Bosonnet, ${ }^{\mathrm{A}}$ Emily Savundra ${ }^{\mathrm{A}}$ and Hakan Kabatas ${ }^{\mathrm{A}}$
}

\begin{abstract}
Aims
To provide virtual triage and, where appropriate, virtual coordination of investigations and care of gastroenterology general practitioner (GP) referrals.

\section{Methods}

A bespoke secure platform was created to receive gastroenterology referrals from GPs from Barking, Redbridge and Havering clinical commissioning groups. Any gastroenterology referral was accepted, excluding urgent or 2-week wait referrals. To support local consultant capacity, we adopted a unique approach by recruiting NHS gastroenterology consultants from around the country. They were able to review gastroenterology referrals via the platform and work in their own time to meet the demand of the area.

All referrals were initially triaged by a gastroenterology consultant. The consultant could triage the patient to GP advice only (discharge), virtual hospital management or refer to hospital outpatient clinic for face-to-face consultation. Virtual hospital management involved a combination of investigations or telephone consultations by junior doctors. All the results were reviewed by the consultant before deciding if the patient could be managed entirely virtually before discharge to the GP, or would require a referral to hospital outpatient clinic, for example, for chronic disease management.
\end{abstract}

\section{Results}

A total of 1,189 of patients were referred to the service from March 2017-January 2018. Of these, $63.83 \%$ entered the virtual hospital pathway, $21.19 \%$ were discharged to GP with advice and $14.97 \%$ were referred to outpatient clinic. The average time taken for the initial consultant triage was 5 hours and 2 minutes compared with several weeks for an initial outpatient appointment. 2.5\% of routine referrals were identified as urgent and $1 \%$ of routine referrals were identified as 2-week wait. No clinically adverse incidents have been reported.

\section{Conclusion}

The virtual hospital has created a safe and efficient healthcare pathway, utilising technology that significantly reduces hospital

Authors: ${ }^{\mathrm{A}}$ Medefer, London, UK; ${ }^{\mathrm{B}}$ Gastroenterology, Medefer, London, UK footfall, reducing patient waiting times and delivers systembased cost savings. We have identified a sustainable solution to improve and meet increasing demand despite limited capacity within the NHS. The virtual hospital demonstrates that majority of patients under virtual specialist care can be managed within the community setting or virtually. Early triage also helps reduce clinical risk for patients who would have otherwise waited several weeks for a clinic appointment.

\section{Conflict of interest statement}

The authors are employed by the service provider. 